\title{
Coagulation and thrombotic risk in thalassemia intermedia
}

\author{
A.T. Taher, K.M. Musallam \\ Department of Internal Medicine, Hematology-Oncology Division, American University of Beirut \\ Medical Centre, Beirut, Lebanon
}

\begin{abstract}
As the life expectancy of $\beta$-thalassemia patients has markedly improved over the last few decades, several manifestations are increasingly recognized. The presence of a high incidence of thromboembolic events, mainly in thalassemia intermedia patients, has led to the identification of a hypercoagulable state in thalassemia. In this review, the current clinical experience attributed to the coagulopathy in thalassemia intermedia patients is summarized. Recommendations for thrombosis prophylaxis are also discussed.
\end{abstract}

\section{Introduction}

The term $\beta$-thalassemia intermedia (TI) was first suggested to describe patients who have a milder anemia compared to patients with $\beta$-thalassemia major (TM), and who usually present to medical attention later in childhood, and remain largely transfusion-independent. However, recent evidence suggests that the diagnosis of $\beta$-thalassemia intermedia (TI) carries higher morbidity than previously recognized, especially in the transfusion-independent patient where the mechanism of disease remains largely unbalanced. ${ }^{1}$ Three main factors highlight the pathophysiology of TI, ineffective erythropoiesis, chronic ane$\mathrm{mia} /$ hemolysis, and iron overload secondary to increased intestinal absorption. ${ }^{1}$ Among the medical complications of TI that were found to occur at high rates, even more frequent than patients with TM, are

Correspondence: A.T. Taher, Professor of Medicine Hematology-Oncology Associate Chair, Research Department of Internal Medicine, American University of Beirut Medical Center, Beirut, Lebanon

Tel. +961.1.350000 - Fax: +961.1.370814. E-mail: ataher@aub.edu.lb

Key words: thalassemia intermedia; hypercoagulability; thromboembolism; stroke; splenectomy; transfusion.

() Copyright A.T. Taher and K.M. Musallam, 2011

Licensee PAGEPress, Italy

Thalassemia Reports 2011; 1(s2):e15

doi:10.4081/thal.2011.s2.e15

This article is distributed under the terms of the Creative Commons Attribution Noncommercial License (by-nc 3.0) which permits any noncommercial use, distribution, and reproduction in any medium, provided the original author(s) and source are credited.

Parts of this work were presented at the "12th International Conference on Thalassemia and Hemoglobinopathies", Antalya (Turkey), 11-14 May 2011. thromboembolic events (TEE), attributed to a state of hypercoagulability (Table 1). ${ }^{1-3}$ We herein review current evidence on TEE in TI patients.

\section{Clinical experience}

Borgna-Pignatti et al. surveyed nine Italian pediatric thalassemia centers, observing that $4 \%$ of the 683 patients with TM and $9.6 \%$ of the 52 patients with TI had experienced a TEE. ${ }^{4}$ The same group showed six years later that $1.1 \%$ of 720 patients with TM in seven Italian centers had thrombosis. ${ }^{5}$ Cappellini et al. followed-up 83 patients with TI over 10 years, 82 of whom were splenectomized, and found that $29 \%$ (24/83) experienced a venous TEE. ${ }^{6}$ One study directly implicated TEE as the cause of death in $2.5 \%$ of transfusion-dependent thalassemia patients. $^{7}$ After examining data from 8,860 patients in the Mediterranean area and Iran, Taher et al. observed that TEE occurred 4.38 times (95\% confidence interval $[\mathrm{CI}] 3.14-6.10, P<0.001)$ more frequently in TI than TM, with more venous events occurring in TI and more arterial events occurring in TM. ${ }^{8}$ It was found that $14 \%$ of mortalities in the whole group were due to TEE. Age above 20 years, splenectomy, family history of TEE, and previous TEE were identified as the main risk factors for thrombosis in TI. Furthermore, the study showed that $68 \%$ of TI patients that had a TEE had an average hemoglobin level of $<9 \mathrm{~g} / \mathrm{dl}$ and only $33 \%$ were receiving regular blood transfusions, whereas $94 \%$ were splenectomized. Moreover, patients receiving aspirin therapy had a significantly lower rate of recurrent TEE. ${ }^{8}$

The evidence for brain involvement in thalassemia dates back to 1972 where $20 \%$ of 138 TM patients in Greece were found to have neurological deficits compatible with transient ischemic attacks (TIAs). ${ }^{9}$ Further evidence of TIAs causing neurological symptoms, such as headaches, hemiparesis, and seizures was shown in $2.2 \%$ of patients with TM in Italy. ${ }^{4}$ Although overt stroke occurs more frequently in TM than TI ( $28 \%$ vs. $9 \%$, respectively), ${ }^{8}$ it has been shown that as many as $37.5 \%$ of patients with TI have asymptomatic brain damage on magnetic resonance imaging (MRI). ${ }^{9}$ A more recent study on Lebanese patients determined that splenectomized adults with TI show a rate of silent white matter lesions as high as $60 \% .{ }^{10}$ The occurrence and multiplicity of the lesions were associated with older age (mean age of 36.1 years for lesion positive-patients vs. 26.1 years for lesion-negative patients) and transfusion naivety (83.3\% of lesionpositive patients have never had a transfusion vs. $25 \%$ of lesion-negative patients) [10]. Another study from Iran followed to confirm these findings ${ }^{11}$

In order to obtain much needed clinical data on the optimal management of patients with TI, the Overview on Practices in Thalassemia Intermedia Management Aiming for Lowering Complication rates Across a Region of Endemicity (OPTIMAL CARE) study evaluated 584 patients with TI at six comprehensive care centers (Lebanon, Italy, Iran, Egypt, United Arab Emirates, and Oman) for 
Table 1. Factors contributing to hypercoagulability in thalassemia intermedia. ${ }^{1}$

\begin{tabular}{ll} 
Factor & Mechanisms \\
Red blood cells & - Formation of reactive oxygen species \\
& - Expression of negatively charged phospholipids \\
& - Enhanced cohesiveness and aggregability \\
Platelets & - Increased platelet aggregation \\
& - Presence of platelet morphologic abnormalities \\
\hline Peripheral blood elements & - Expression of endothelial adhesion molecules and tissue factor on endothelial cells \\
- Formation of microparticles \\
- High platelet counts and hyperactivity \\
- High levels of negatively charged red blood cells \\
Thitric oxide & - Decreased levels leading to vasoconstriction \\
Thrombophilia & - Decreased levels of antithrombin III, protein C and protein S \\
& - No role for prothrombotic mutations \\
\hline Other factors & - Hardiac dysfunction \\
& - Hepatic dysfunction \\
\hline
\end{tabular}

the associations between patient and disease characteristics, treatment received, and the rate of clinical complications. ${ }^{12}$ Thrombosis was the $5^{\text {th }}$ most common complication, affecting $14 \%$ of the patient population. On multivariate analysis, splenectomy, age above 35 years, and a serum ferritin level $\geq 1000 \mu \mathrm{g} / \mathrm{l}$ were associated with a higher risk for thrombosis. ${ }^{12}$ Conversely, a positive history of transfusion and a hemoglobin level $\geq 9 \mathrm{~g} / \mathrm{dl}$ were found to be protective against thrombosis $^{12}$ (Table 2). A higher occurrence of TEE with advancing age was also observed. ${ }^{13}$

A sub-study of the OPTIMAL CARE examined the characteristics of splenectomized patients with TI who develop TEE aiming to identify high-risk patients who deserve further consideration for preventive strategies. ${ }^{14}$ Splenectomized patients with documented TEE (Group I, $n=73$ ) were age- and sex-matched to splenectomized patients without TEE (Group II) and non-splenectomized patients without TEE (Group III). The study determined that splenectomized TI patients who experience TEE are characterized by high nucleated RBC ( $\geq 300 \mathrm{x}$ $10^{6} /$ ) and platelet counts ( $\left.\geq 500 \times 10^{9} /\right)$ ), are more likely to have evidence of pulmonary hypertension (PHT), and be transfusion naive. As such, it was suggested that splenectomized TI patients at risk of developing TEE may be identified early on by these laboratory markers, presence of PHT, and transfusion status. ${ }^{14}$ The study further examined how long it took for a TEE to develop following splenectomy and found the median time to thrombosis to be 8 years. ${ }^{14}$ This delay indicates that TEE in splenectomized patients with TI is not an acute complication, but a manifestation of a chronic underlying process, further emphasizing the need for a long-term treatment modality for prevention. ${ }^{14}$

\section{Prevention and Management}

Reduction of the proportion of circulating RBCs with thrombogenic potential may be attained by introducing blood transfusions, and may justify the lower rate of TEE in transfused vs. non-transfused patients in previous studies. ${ }^{8}, 10,12,14$ As such, transfusion therapy may be worthwhile to prevent the occurrence of TEE and other complications ${ }^{1}$ in TI patients for whom current practice does not necessarily recommend transfusions. Rather than enforcing the regular transfusion regimens implemented in TM, blood transfusion, if initiated in patients with TI will should be individually tailored to meet patient needs. Although introduction of blood transfusions will increase the rate of iron accumulation, effective methods of iron chelation are now available, and the benefits of transfusion therapy may greatly outweigh the cost and inconvenience of iron chelation therapy. ${ }^{15}$ This approach requires prospective evaluation.

Since splenectomy is a major contributor to TEE in patients with thalassemia, ${ }^{16}$ reassessment of the procedure and appropriate risk benefit-evaluation prior to any attempt at splenectomy is called for. This is also essential in line with recent evidence on high rates of other clinical complications after splenectomy, ${ }^{12}$ alongside the well-known increased susceptibility to infection. ${ }^{17}$

The literature lacks proper evidence on the role of antiplatelet or anticoagulant agents in the management of thalassemia. ${ }^{3}$ The lower recurrence rate of TEE in TI patients, who took aspirin after their first TEE, when compared to those who did not, suggests a potential role for aspirin. ${ }^{8}$ Moreover, the association of higher platelet counts with TEE in patients with TI further suggests a role for aspirin in this patient population. ${ }^{14}$

Table 2. Predictors of thrombosis in the OPTIMAL CARE study. ${ }^{12}$

\begin{tabular}{lcccc} 
Parameter & Adjusted OR & 95\% CI & & \\
Age $>35$ & 2.59 & 1.27 & $1.39-4.87$ & 0.003 \\
Female & 0.41 & $0.74-2.19$ & 0.387 \\
\hline Hemoglobin $\geq 9 \mathrm{~g} / \mathrm{dl}$ & 1.86 & $0.23-0.71$ & 0.001 \\
Ferritin $\geq 1000 \mu \mathrm{g} / \mathrm{L}$ & 6.59 & $1.09-3.16$ & 0.023 \\
\hline Splenectomy & 0.28 & $3.09-14.05$ & $<0.001$ \\
\hline Transfusion & 0.56 & $0.16-0.48$ & 0.001 \\
Hydroxyurea & 0.97 & $0.28-1.10$ & 0.090 \\
\hline Iron chelation & & $0.56-1.68$ & 0.912 \\
\hline
\end{tabular}


Fetal hemoglobin inducing agents like decitabine and hydroxycarbamide were also shown to lower plasma markers of thrombin generation. ${ }^{2}$ Hydroxycarbamide may modulate hypercoagulability in several ways, it may reduce phospholipid expression on the surface of RBCs and platelets, and decrease RBC adhesion to thrombospondin, a thrombin sensitive protein. ${ }^{2}$ It may also decrease leukocyte count, particularly monocytes expressing transcription factor, in addition to being a nitric oxide donor. ${ }^{18}$

It is recommended that each patient be assessed individually and assigned a personalized thrombotic risk based on intrinsic and extrinsic factors. High nucleated RBC and platelet counts, evidence of PHT, and transfusion naivety can be used as indicators of TEE for splenectomized patients with TI and could be practical in the clinical setting. ${ }^{14}$ Such a risk-assessment model would be valuable in identifying highrisk patients and targeting them for further testing. Several diagnostic tests are being explored to help identify patients at risk, with promising preliminary results. ${ }^{19}$

The hypercoagulable state in TI is due to multiple elements, a combination of which is often the drive behind a clinical TEE. Splenectomy and transfusion naivety are increasingly highlighted as important risk factors for TEE. An individualized approach is recommended to establish an optimal strategy for preventing the occurrence of this complication of TI.

\section{References}

1. Taher AT, Musallam KM, Cappellini MD, Weatherall DJ. Optimal management of $\beta$ thalassaemia intermedia. $\mathrm{Br} \mathrm{J}$ Haematol. 2011;152:512-23.

2. Cappellini MD, Motta I, Musallam KM, Taher AT. Redefining thalassemia as a hypercoagulable state. Ann N Y Acad Sci. 2010;1202:231-6.

3. Taher AT, Otrock ZK, Uthman I, Cappellini MD. Thalassemia and hypercoagulability. Blood Rev. 2008;22:283-92.

4. Borgna Pignatti C, Carnelli V, Caruso V, et al. Thromboembolic events in $\beta$ thalassemia major: an Italian multicenter study. Acta Haematol. 1998;99:76-9.

5. Borgna-Pignatti C, Rugolotto S, De Stefano P, et al. Survival and complications in patients with thalassemia major treated with transfusion and deferoxamine. Haematologica. 2004;89:1187-93.

6. Cappellini MD, Robbiolo L, Bottasso BM, Coppola R, Fiorelli G, Mannucci AP. Venous thromboembolism and hypercoagulability in splenectomized patients with thalassaemia intermedia. Br $\mathrm{J}$ Haematol. 2000;111:467-73.

7. Zurlo MG, De Stefano P, Borgna-Pignatti C, et al. Survival and causes of death in thalassaemia major. Lancet. 1989;2:27-30.

8. Taher A, Ismaeel H, Mehio G, et al. Prevalence of thromboembolic events among 8,860 patients with thalassaemia major and intermedia in the Mediterranean area and Iran. Thromb Haemost. 2006;96:488-91.

9. Manfre L, Giarratano E, Maggio A, Banco A, Vaccaro G, Lagalla R. MR imaging of the brain: findings in asymptomatic patients with thalassemia intermedia and sickle cell-thalassemia disease. AJR Am J Roentgenol. 1999;173:1477-80.

10. Taher AT, Musallam KM, Nasreddine W, Hourani R, Inati A, Beydoun A. Asymptomatic brain magnetic resonance imaging abnormalities in splenectomized adults with thalassemia intermedia. J Thromb Haemost. 2010;8:54-9.

11. Karimi M, Bagheri H, Rastgu F, Rachmilewitz EA. Magnetic resonance imaging to determine the incidence of brain ischaemia in patients with $\beta$-thalassaemia intermedia. Thromb Haemost. 2010;103:989-93.

12. Taher AT, Musallam KM, Karimi M, et al. Overview on practices in thalassemia intermedia management aiming for lowering complication rates across a region of endemicity: the OPTIMAL CARE study. Blood. 2010;115:1886-92.

13. Taher AT, Musallam KM, El-Beshlawy A, et al. Age-related complications in treatment-naive patients with thalassaemia intermedia. $\mathrm{Br}$ J Haematol. 2010;150:486-9.

14. Taher AT, Musallam KM, Karimi M, et al. Splenectomy and thrombosis: the case of thalassemia intermedia. J Thromb Haemost. 2010;8:2152-8.

15. Taher A, Hershko C, Cappellini MD. Iron overload in thalassaemia intermedia: reassessment of iron chelation strategies. $\mathrm{Br} \mathrm{J}$ Haematol. 2009;147:634-40.

16. Crary SE, Buchanan GR. Vascular complications after splenectomy for hematologic disorders. Blood. 2009;114:2861-8.

17. Cadili A, de Gara C. Complications of splenectomy. Am J Med. 2008;121:371-5.

18. Sumiyoshi A, Thakerngpol K, Sonakul D. Pulmonary microthromboemboli in thalassemic cases. Southeast Asian J Trop Med Public Health. 1992;23:29-31.

19. Tripodi A, Cappellini MD, Chantarangkul V, et al. Hypercoagulability in splenectomized thalassemic patients detected by whole-blood thromboelastometry, but not by thrombin generation in plateletpoor plasma. Haematologica. 2009;94:1520-7. 